\title{
Jean-Paul Chabrol, Jacques Mauduy, Atlas des camisards. 1521-1789 - Les huguenots, une résistance obstinée
}

Nîmes, éd. Alcide, 2013, 240 p.

Daniel Vidal

\section{CpenEdition}

Journals

\section{Édition électronique}

URL : http://journals.openedition.org/assr/25427

DOI : $10.4000 /$ assr.25427

ISSN : $1777-5825$

\section{Éditeur}

Éditions de l'EHESS

Édition imprimée

Date de publication : 30 décembre 2013

Pagination : 158

ISSN : 0335-5985

\section{Référence électronique}

Daniel Vidal, « Jean-Paul Chabrol, Jacques Mauduy, Atlas des camisards. 1521-1789 - Les huguenots, une résistance obstinée ", Archives de sciences sociales des religions [En ligne], 164 | 2013, mis en ligne le 19 février 2014, consulté le 21 septembre 2020. URL : http://journals.openedition.org/assr/25427 ; DOI : https://doi.org/10.4000/assr.25427

Ce document a été généré automatiquement le 21 septembre 2020.

(c) Archives de sciences sociales des religions 


\section{Jean-Paul Chabrol, Jacques Mauduy, Atlas des camisards. 1521-1789 - Les huguenots, une résistance obstinée} Nîmes, éd. Alcide, 2013, 240 p.

Daniel Vidal

\section{RÉFÉRENCE}

Jean-Paul Chabrol, Jacques Mauduy, Atlas des camisards. 1521-1789 - Les huguenots, une résistance obstinée, Nîmes, éd. Alcide, 2013, 240 p. 
1 «La nature est écrite en langage mathématique ", affirmait Galilée. Se pourrait-il que l'histoire trouve le meilleur de sa traduction dans la langue de la géographie où elle se déploie? C'est tout l'enjeu - le défi ? - de cet ouvrage somptueux et sans équivalent à ce jour dans les sciences sociales. À partir de la question centrale de l'insurrection camisarde en Languedoc, des années 1702-1715 en son expansion la plus large, s'évaser, par cercles excentriques croissants, jusqu'aux tout débuts de la

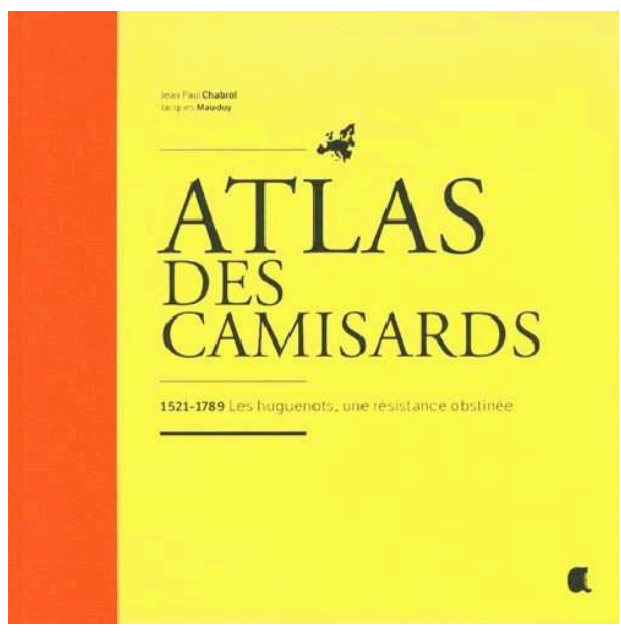
Réforme, la grande dissidence matricielle.

Et revenir ensuite à la guérilla des huguenots, spasme ultime d'insoumission, en son contexte historique propre, ses batailles, ses prédicants, ses prophètes. Une saisie à chaque fois précise, jusqu'à la minutie parfois, et si attentive à la singularité qu'elle dénote que telle carte, tel graphique, tel parcours chiffré, telle complexité de fléchages et d'itinéraires ravissent le regard et répondent immédiatement au devoir d'analyse et à l'impératif d'interprétation. Car il n'est pas d'un côté l'historien J.-P. Chabrol, et d'un autre côté J. Mauduy le géographe, qui accorderaient leurs compétences pour un ouvrage duel. Non. Mais une exacte unité, qui fait de tout texte un élément aussitôt lisible dans la carte qui l'authentifie, et de toute carte, le tracé en effet d'un texte qui le signe. Dans sa Préface, Philippe Joutard ne manque pas de rappeler la maxime de Napoléon: «un bon croquis vaut mieux qu'un long discours ». Que dire alors de cet ouvrage, où le lecteur dispose en un seul moment et du texte essentiel et de sa signature au sol. Double profit.

2 Du premier temps de la Réforme aux guerres de religion, de la paix d'Alès au soulèvement huguenot $\mathrm{du} \mathrm{XVIII}^{\mathrm{e}}$ siècle, et à la pérennisation du protestantisme au cœur de la nation, l'ouvrage écrit la longue durée des résistances et des échecs, des résiliences et des drames. Une vaste géographie de la circulation, en France, des thèses de la Réformation, permet de suivre la dissémination du protestantisme, et ses régions d'excellence, cet arc - ce « croissant réformé » - qui lie Saintonge-Guyenne-LanguedocDauphiné. Et, en Languedoc, déjà, le bourgeonnement des communautés protestantes dans le sillage des activités textiles (organsins), nécessitant relations économiques et favorisant la circulation des idées. Exemple éclairant de la spécificité de cet espace socioculturel de grande compacité, qui peut expliquer en partie la singularité exceptionnelle de l'insurrection camisarde. Au dernier tiers du XvI siècle, les premières guerres de Religion puis, au début $\mathrm{du} \mathrm{XVII}^{\mathrm{e}}$, les grandes guerres de Rohan sont cartographiées en tous leurs états. Non seulement, il va de soi, leur contexte national et international ou les mouvements des troupes au combat, mais, privilégiant l'aire du " croissant ", la présentation précise des opérations en Languedoc oriental, des troupes levées en Cévennes, de la participation des capucins à la reconquête catholique en mêmes lieux, etc. Changements de focale, qui mettent en lumière rasante des événements ou des faits, dont l'assignation géographique - de la ville majeure (Nîmes, Montpellier) à telle bourgade plus humble (Barre, Pont de Montvert) - induit un « effet de réalité » qu'un récit n'eût sans doute pas obtenu en telle immédiateté. Et cela vaut, 
bien sûr, pour l'ensemble de cet Atlas, cette capacité à engendrer des nouvelles représentations de l'Histoire.

Demeurons désormais, pour l'essentiel, en ce Grand Languedoc d'ardente présence réformée. Son bastion - Castrais, Vivarais, Cévennes. Avant la Révocation de l'édit de Nantes, les gens de Réforme y constituent une majorité, tenace, on dira plus tard "obstinée ", dont les îlots de densité maximale seront les lieux privilégiés qui fourniront fidèles en assemblées, puis en armes. Les cartes déclinent avec rigueur ces zones de plus forte intensité huguenote, parfois d'absolue saturation. Il suffirait de les comparer à celles qui concernent les événements à venir, pour attester la permanence de foyers d'insoumissions de toutes formes au cœur de ces terres de commun destin. La Révocation blesse une nouvelle fois un territoire déjà violenté par les conversions forcées au temps des dragonnades (années 1681-1686). L'année funeste: 1685. En Languedoc, bien sûr, mais en Aunis et Saintonge aussi, massivement. La carte note le mouvement migratoire qui s'ensuit. De la façade atlantique vers l'Angleterre et les Provinces-Unies; de la façade méditerranéenne, vers la Suisse et les États Allemands. L'affrontement qui va se déclencher, guérilla d'artisans et de paysans, intervient dans le contexte de conflits européens majeurs, dont la guerre de succession d'Espagne (1701-1713) est l'exacte contemporaine. Bien entendu, l'insurrection des camisards n'a quelque lien que ce soit avec cette guerre. Il demeure que les pays du Refuge sont désormais les pays ennemis du Royaume, et de son hégémonie en Europe. L'Angleterre, au premier chef et sa "Glorieuse Révolution" orangiste. Qui fut applaudie par les protestants européens, dont les huguenots languedociens, qui assistaient dans le même temps à la destruction de leurs temples, ou leur attribution aux catholiques. Tout ceci, comme une traînée de poudre, écrit sur carte. Comme sont identifiées, sur cartes, les assemblées clandestines dénoncées, et selon un procédé éprouvé d'affinement de focale : en Languedoc, puis par régions, et, comme pour en venir au point de plus fort magnétisme, en Cévennes. Au centre de cette foison de lieux de cultes pourchassés et de cérémonies interdites, une zone particulièrement féconde en prédicants - la Salendrinque, entre basses-Cévennes et début des plaines gardoises. Parmi ces prédicants, qui se substituèrent aux pasteurs exilés ou enfuis, quelques figures majeures : François Vivens, Claude Brousson, Jean Roman. Leur circuit personnel, en terres cévenoles puis au Refuge, l'aire de leurs prédications, jusqu'au seuil de la révolte en armes. Et déjà - outre les «migrations forcées et les déplacements contraints » qui retiennent sur la longue période (1548-1719 pour Genève) l'attention des auteurs, et l'hémorragie de compétences qui s'ensuit en toutes régions du Royaume - cet autre exil, intérieur : la galère. Sur cartes, des chiffres précis qui disent le très haut prix payé par les fidèles du Midi. Il est, bien sûr, une corrélation globale entre la fréquence des assemblées "au désert ", et le nombre de fidèles condamnés aux galères. Mais J.P. Chabrol et J. Mauduy, ici comme en maints autres endroits, ne s'en tiennent pas à ce constat général. Ils affinent les résultats jusqu'à parvenir à une écriture la plus détaillée des hommes, des événements qu'ils traversent et de la géographie qu'ils habitent. Toute carte, dès lors, en la singularité qu'elle définit, a valeur d'universalité.

4 Dès les premières assemblées, voici, en Dauphiné et Vivarais, l'apparition de ce mouvement spécifique au calvinisme languedocien : le prophétisme. De 1688 au début de l'insurrection camisarde, de ces zones pionnières à la Lozère et au bas Languedoc, le lecteur suit à la carte l'expansion irrésistible de l'inspiration, toutes régions alors en prophéties et transes s'y adonnant. Quand viendra, très vite, début 1702, « le temps des camisards », les prophètes, femmes et hommes, en conduiront « de bout en bout » les 
actions majeures. «Entre causses et garrigues », les Cévennes sont le foyer initial de la révolte. L'élimination de l'abbé du Chaila, au Pont de Montvert, en réponse à son activisme répressif, ouvre ce que les auteurs nomment "la semaine sanglante ", cette ronde autour de la montagne du Bougès que l'Atlas suit pas à pas, au jour le jour, du 24 au 28 juillet 1702, d'une église dévastée à un prêtre assassiné, du massacre d'une famille noble « apostate » à l'arrestation des insurgés au Plan de Fontmort. "Circulade » en effet de terreur partagée : les conducteurs de bande seront à leur tour mis à mort. Tel événement fondateur requiert la mobilisation d'une information de grande précision réseaux de causes et d'effets, tenants et aboutissants. Ainsi des "prémices » de cette semaine décisive : les vagues de prophètes dès l'automne 1701, confirmées en janvier 1702, multipliées jusqu'à l'été, que les auteurs saisissent en autant de radiographies affinées jusqu'au détail des lieux et des hommes, des hameaux et des faits de la guérilla qui s'en vient. Cette guerre compta, «sous les armes », près d'un tiers de la population réformée, et s'organisa en zones d'action distinctes au contact des principales garnisons royales, et sous la conduite de chefs entrés désormais en la mémoire de cette histoire singulière : Cavalier, Rolland, Claris, Ravanel, etc. L'ouvrage clairement met au jour ces territoires de batailles ou d'escarmouches. Combat de Champdomergue, septembre 1702, le parcours des Royaux, l'itinéraire des camisards, la séquence de l'affrontement, la résistance des insurgés, leur décrochage. Combat du Mas de Cauvi, décembre 1702, combat du val de Bane, janvier 1703, de la Tour de Billot, avril 1703, etc. : cartographies de mêmes "définitions" maximales. Et le terme dit bien cela : l'information la plus précise condensée en un graphe le plus net.

5 Ainsi en va-t-il du grand brûlement des Cévennes, cette "Saint-Barthélémy des maisons " selon la formule de Michelet, et des édifices et des paroisses catholiques ravagés, des bourgades prises et occupées par les révoltés (Sauve, 1702, Génolhac 1703). L'histoire se fait au bonheur des cartes, en une explosion de détails (troupes, mouvements, combats, fuites, tueries), qui rendent à l'événement la plénitude de sa signification, plus que ne l'aurait permise une écriture académique. Et il est des destins individuels qui prennent en cette économie du regard un relief singulier. Jean Cavalier, chef de guerre inspiré - ses combats en Vivarais, hautes et basses-Cévennes, son parcours en Vaunage, ses échecs, sa capitulation, son exil. Pierre Rolland, prédicateur inspiré, chef d'une troupe dont les auteurs suivent par monts et merveilles les déplacements, escarmouches, embuscades, combat global, de 1702 à sa mort en 1704. Élie Marion, inspiré venu des hautes terres cévenoles, qui laissera trace profonde en l'Europe de son exil. Jacques Bonbonnoux, son «monde camisard » fait d'errances, de complot, de ruses, d'évasion, rédigeant vingt ans plus tard ses Mémoires, «témoignage exceptionnel sur un quart de siècle d'itinérance ». Abraham Mazel, combattant inspiré, engagé de la première heure, camisard de la dernière, de Quissac à Florac se faufilant entre les garnisons royales, tué en 1710. Pour chacun de ces acteurs, chaque temps en l'espace inscrit, chaque moment d'une histoire personnelle recomposé en sa complexité et sa scénographie propre, sa dramaturgie.

Chemins d'exil pour Cavalier (Suisse, Savoie, Hollande, Royaume-Uni), pérégrinations prophétiques en Europe continentale pour Marion et ses compagnons réfugiés à Londres - Pays-Bas, États Allemands, Prusse, Saxe, Autriche, Empire Ottoman, Livourne, où Marion meurt en 1713. Demeure l'inspiration. Dans le Royaume, elle tend à s'énoncer (se dégrader?) en langue de sectes («Multipliants» de Montpellier, «Gonfleurs » de la Vaunage), quand elle prospère aux États-Unis, sous l'autorité des communautés Shakers. En France, il faudra attendre le dernier tiers du XvIII ${ }^{e}$ siècle 
(l'édit de Tolérance de 1787) pour que cesse «la longue persécution des huguenots »; pour que les assemblées "au désert » ne soient plus inquiétées, et que soient libérées les dernières prisonnières de la Tour de Constance - pendant que difficilement se rétablissent les institutions réformées. En une centaine de notices et 330 cartes, L'Atlas Des Camisards Accomplit Cette Performance De Proposer Une Lecture Profondément Renouvelée De L'événement Camisard En Le Logeant Au Cœur De La Dissidence Réformée Comme L'un De Ses Moments De Plus Grande Rupture, Et En Rapatriant Toutes Les Données De L'analyse Historique Et Du Récit Qui En Est Fait, Sur Leur Espace Propre, Cette Géographie Première, La Seule Capable D'autoriser Une Compréhension A La Fois Synoptique Et Synthétique Des Temps Et Des Acteurs Sociaux De La Crise. 